\title{
Chemical Pathology
}

\section{LIQUID CHROMATOGRAPHY MASS SPECTROMETRY OF 17-HYDROXYPROGESTERONE TO IMPROVE SCREENING FOR CONGENITAL ADRENAL HYPERPLASIA IN NEW ZEALAND}

Mark de Hora

Chemical Pathology, LabPlus, Auckland, New Zealand

Aim: To develop a liquid chromatography mass spectrometry (LCMS) method for blood spot 17-hydroxyprogesterone (17OHP) as a second tier newborn screening test for congenital adrenal hyperplasia $(\mathrm{CAH})$.

To determine whether the method will improve screening for $\mathrm{CAH}$ in New Zealand

Method: The LCMS method was developed by eluting 17OHP from blood spots using $100 \%$ methanol with added deuterated internal standard. 17OHP was extracted by injection onto an inline solid phase extraction column before back-flushing with elution solvent onto an analytical column and separation from potentially interfering compounds before mass spectrometry detection.

Results: Analysis of 17OHP on 148 samples from patients treated for $\mathrm{CAH}$ yielded a regression line $\mathrm{y}=0.76 \mathrm{x}+4.79$ and $\mathrm{r}^{2}=0.81$ when compared with the current method. The coefficient of variation $(\mathrm{CV})$ was $25 \%(40-150 \mathrm{nmol} / \mathrm{L})$. Recovery was $92-108 \%$ and the linearity up to $2000 \mathrm{nmol} / \mathrm{L}$.

Of 62 initially positive CAH screens, 21 remained elevated after measurement with immunoassay after solvent extraction. Analysis by LCMS resulted in 7 positive screens, thus reducing the false positive rate by $67 \%$.

Conclusions: Initial results demonstrate the potential for improved specificity for screening by adopting an LCMS assay for 17-OHP. Precision and accuracy improvements will require an instrument with higher sensitivity.

\section{UNUSUAL CAUSE OF PSEUDOHYPERKALAEMIA IN PRIMARY CARE CLINIC}

Vanessa Man Har Lo

Clinical Pathology Laboratory, Department of Pathology, Hong Kong Sanatorium and Hospital, Hong Kong Special

Administrative Region

Backgrounds: Our hospital has three primary care clinics (PCC1, 2 and 3) outside the main campus. Blood sampled in PCC were sent to the hospital laboratory for analysis with transportation time 2-6 hours. PCC1 accounted for the highest rate of pseudohyperkalaemia, $21 \%$, while the rest were below $5 \%$. Root cause identification followed by constructive solution is urgently required to avoid unnecessary and potentially detrimental therapy due to pseudohyperkalaemia.

Method: From 3 October to 28 November 2013 systematic root cause analysis was conducted by simulation study focussing on preanalytical procedures including: (1) blood sampling technique, (2) sample preparation procedure while sitting in PCC waiting for courier to pick up, (3) transportation conditions including temperature and turbulence effect leading to red cell sediments remixed with plasma during speedy driving, and (4) potassium stability in gel separated plasma up to 9 hours. Statistical analysis tools Deming regression and Bland and Altman plot were adopted.

Results: Excessive fist clenching was identified to be the culprit. Pseudohyperkalaemia was recorded in 10 out of 16 study cases. Upon elimination of the inappropriate procedure, up to 22 September 2015 the occurrence rate of pseudohyperkalaemia in PCC1 dropped dramatically to $7 \%$, comparable with those in $\mathrm{PCC} 2$ and $\mathrm{PCC} 3$.

\section{A CASE OF EXTREME HYPONATRAEMIA SECONDARY TO A LOW SOLUTE DIET AND PRIMARY POLYDIPSIA}

\section{J.D. Smith ${ }^{1}$, L. Roberts ${ }^{2}$, H.G. Schneider}

${ }^{1}$ Clinical Biochemistry Unit, Alfred Pathology Services, Alfred Health, Melbourne, and ${ }^{2}$ Intensive Care Unit, Alfred Health, Melbourne, Vic, Australia

Introduction: Hyponatraemia is the most common electrolyte disorder in hospitalised patients. Aetiologies commonly encountered include iatrogenic (e.g., impaired free water clearance due to thiazide diuretics) and the syndrome of inappropriate ADH secretion (SIADH). Hyponatraemia secondary to impaired free water clearance due to low solute intake is relatively uncommon. Classic cases have been described in alcoholics ('beer potomania') or patients on a 'tea and toast diet'. We report a case of severe hyponatraemia (sodium $94 \mathrm{mmol} / \mathrm{L}$ ) in the setting of a low solute diet and primary polydipsia caused by ibuprofencodeine dependence.

Clinical features: A 30-year-old male was brought in to the emergency department at tertiary referral centre following a witnessed tonic-clonic seizure. He had no relevant past medical or psychiatric history but had developed a dependence on over-the-counter pain medication and was regularly taking ibuprofen-codeine $(200 \mathrm{mg} / 12.8 \mathrm{mg})$, up to 20 tablets daily. Associated nausea and abdominal pain in this setting resulted in a severely restricted diet of small amounts of rice and toast, and an associated dry mouth resulted in a daily water intake of up to 5 litres. On presentation, plasma sodium was $94 \mathrm{mmol} / \mathrm{L}$ with serum osmolality $207 \mathrm{mOsm} / \mathrm{kg}$. Malnutrition was evidenced by a low BMI (14), low albumin and a microcytic anaemia with a low ferritin $(8 \mu \mathrm{g} / \mathrm{L})$ consistent with iron deficiency.

Progress: Initial treatment was with hypertonic (3\%) saline as a bolus followed by normal saline infusion in ICU. This solute load resulted in a urine output of $800 \mathrm{~mL} /$ hour with correction of plasma sodium to $112 \mathrm{mmol} / \mathrm{L}$ in 10 hours. DDAVP and $5 \%$ dextrose were administered to slow the rate of correction. Plasma sodium was corrected slowly within the next few days. When discharged to the ward he exhibited some ataxia, dysarthria and behavioural disturbance and osmotic demyelination syndrome 
was suspected. Magnetic resonance imaging of the brain was normal and he made a good neurological recovery.

Conclusion: Hyponatraemia due to a low solute diet and impaired free water excretion is uncommon. Treatment of this type of hyponatraemia with a solute load can result in a rapid correction of plasma sodium concentration, placing patients at risk of the osmotic demyelination syndrome.

\section{A TALE OF LOCUST SWARMS, CANNIBALS, AGEING AND HUMAN OBESITY}

Stephen J. Simpson

Charles Perkins Centre, The University of Sydney, Sydney, NSW, Australia

There can be few more important challenges in modern biology than explaining how the phenotypic features of organisms contribute to the populations, communities and ecosystems within which they exist, and how these in turn respond to changing environmental conditions. The phenotype sits at the confluence of influences arising from the genes and the environment and it is the phenotype which is subject to natural selection. I will begin with my group's work on locusts and other insects to show how spanning individuals to ecosystems can be achieved by combining theory, laboratory and field experiments and by using techniques from a range of disciplines, including molecular biology, neurophysiology, biochemistry, behaviour, mathematics, statistical physics, computer science, engineering, evolutionary theory and landscape ecology. Locust plagues are one of the most infamous insect scourges, affecting the lives of 1 in 10 people on the planet. While of interest in their own right, locusts have also provided important new clues into the causes of human obesity and metabolic disease, how we age, and the complex behaviour of crowds. Many of these insights have arisen through a new understanding of the role of nutrition in shaping phenotypic plasticity. I will introduce the principles of nutritional geometry, which arose initially from studies on locusts, and show how using this powerful conceptual framework has yielded fundamental new understanding about the human obesity epidemic and the nutritional determinants of reproduction, aging and lifespan.

\section{THE ABORIGINAL HUNTER-GATHERER LIFESTYLE: LESSONS FOR CHRONIC DISEASE PREVENTION}

\section{Kerin O'Dea}

School of Population Health, University of South Australia, Adelaide, SA, Australia

In 1982 when a group of middle-aged Aboriginal people with type 2 diabetes reverted to their traditional hunter-gatherer lifestyle for just 7 weeks, all of the metabolic abnormalities of diabetes were greatly ameliorated or reversed, as were the major risk factors for cardiovascular disease. Three factors were likely responsible for these striking improvements: low energy intake; high protein, low fat diet; and elevated physical activity. Low energy intake (estimated $1200 \mathrm{kcal} /$ day) was probably the most important.

The most important lesson that can be drawn from this study is that metabolic control in type 2 diabetes can be greatly improved with healthy lifestyle interventions. The diet in the 1982 study was rich in very lean meat from wild animals: its high protein content aided satiety despite low energy intake. The carbohydrate was mostly high fibre and slowly digested. The fat content was low, but relatively rich in long chain highly polyunsaturated fats (omega-3 and omega-6). Recent research has demonstrated that ectopic fat is a driver of both defects of type 2 diabetes: insulin resistance when in the liver, and impaired insulin secretion when in the pancreas. Both are potentially reversible by healthy diets instituted early in the disease process. However, current population trends to increased obesity are driven by the powerful transnational food industry. Preventive interventions will therefore be very challenging.

\section{HUNTING FOR THE SIGNATURES OF CANCER BY PLASMA DNA SEQUENCING}

Rossa W.K. Chiu

Li Ka Shing Institute of Health Sciences and Department of Chemical Pathology, Faculty of Medicine, The Chinese

University of Hong Kong, Hong Kong Special Administrative Region

Liquid biopsy refers to the analysis of DNA released by tumours into the circulation of patients for the detection, prognostication, selection of therapeutic targets or monitoring of malignancies. For example, regulatory approval has been granted for the detection of epidermal growth factor receptor mutations in plasma of patients with non-small cell lung cancers as a means to stratify therapy. However, the key challenge in the diagnostics of cancer is about its early detection. Given that cancers are highly heterogeneous, our group has been developing whole genome approaches to detect the signatures of cancer via a liquid biopsy. The rationale is to extract as much of the molecular information as possible from a plasma sample to catch a glimpse of the possible presence of cancer. Genomewide approaches to detect tumour-associated copy number aberrations, molecular size profile, DNA methylation signatures and transcriptomic features from circulating nucleic acids have been developed. We have further developed an approach to pinpoint the anatomical location of malignancy, naming to determine a tissue map non-invasively. In summary, many facets of molecular information could be extracted from a liquid biopsy.

Acknowledgement: Supported by the Research Grants Council of the Hong Kong SAR Government under the Theme-based research scheme (T12-404/11).

\section{HOW SHOULD WE DEAL WITH MISSED TEST RESULTS AND PENDING RESULTS AT DISCHARGE?}

Andrew Georgiou

Centre for Health Systems and Safety Research, Australian Institute of Health Innovation, Macquarie University, Sydney, NSW, Australia

The failure to follow-up test results is an area of patient safety risk which can have major consequences for the quality of care, potentially leading to missed diagnoses and sub-optimal patient outcomes. Previous research has shown that pathology and imaging test results fail to be followed up in $20-62 \%$ of inpatients, and in up to $75 \%$ of patients treated in an emergency 